\title{
Geodetic Coordinate Systems in Florida: GPS ConTRIBUTION
}

\author{
By Tomás Soler, ${ }^{1}$ William E. Strange, ${ }^{2}$ and Jeffrey S. Ferguson ${ }^{3}$
}

\begin{abstract}
Aastract: Measurements and results derived from the three-dimensional Global Positioning System (GPS) are more accurate than classical geodetic observations and methods used until recently to define conventional horizontal (geometric) datums, e.g., the North American Datum of 1983, the final readjustment of which was finished in 1986, thus the notation NAD 83 (1986). The situation may not be strictly the same with respect to vertical (physical) datums such as the National Geodetic Vertical Datum of 1929 (NGVD 29) because an accurate geoid is required. In this paper comparisons of standard datums [NAD 83 (1986), NGVD 29] with recent GPS results in the state of Florida are analyzed. Their relationship with a very long baseline interferometry (VLBI) reference frame is also discussed. The investigation shows that overall the relative accuracy of NAD 83 (1986) in Florida is between $1 / 200,000$ and $1 / 500,000$ or from five to two parts per million (ppm), yet still not as good as the two-dimensional results derived from GPS.
\end{abstract}

\section{INTRODUCTION}

The Global Positioning System (GPS) project, Florida High Precision Network (HPN), was planned with the intention of establishing throughout the state of Florida an ultraprecise framework of GPS-determined points, to which some marks of the existing horizontal [North American Datum (NAD) 83 (1986)] and vertical [National Geodetic Vertical Datum (NGVD) 29] are connected. The project was jointly sponsored by the National Geodetic Survey (NGS), a division of the National Oceanic and Atmospheric Administration (NOAA), the Florida Department of Natural Resources (FDNR), the Bureau of Aviation of the Florida Department of Transportation (FDOT), and the Federal Aviation Administration (FAA).

The project's primary objective was to implement a statewide network of stations positioned relative to one another with a horizontal relative accuracy of $1: 1,000,000(1 \mathrm{ppm})$ or better. This accomplishment would provide the necessary locations for subsequent densification by geodetic engineers and surveyors, generating the required coordinate grid that will support any future Geographic and Land Information System (GIS/LIS) development in the state (Burton 1989).

This fundamental GPS network includes the point TIMER on the grounds of the Richmond U. S. Naval Observatory (USNO) complex, tied by conventional surveys to the phase center of the fixed very long baseline inter-

${ }^{1}$ Chf., Analysis and Design Sect., Nat. Geodetic Survey, Charting and Geodetic Services, Nat. Ocean Service, Nat. Oceanic and Atmospheric Administration, Rockville, MD 20852.

${ }^{2}$ Chf. Geodesist, Nat. Geodetic Survey, Charting and Geodetic Services, Nat. Ocean Service, Nat. Oceanic and Atmospheric Administration, Rockville, MD.

${ }^{3}$ Lieutenant, NOAA Corps, Space and Physical Geodesy Branch, Nat. Geodetic Survey, Charting and Geodetic Services, Nat. Ocean Service, Nat. Oceanic and Atmospheric Administration, Rockville, MD.

Note. Discussion open until October 1, 1991. To extend the closing date one month, a written request must be filed with the ASCE Manager of Journals. The manuscript for this paper was submitted for review and possible publication on October 18, 1990. This paper is part of the Journal of Surveying Engineering, Vol. 117, No. 2, May, 1991. CASCE, ISSN 0733-9453/91/0002-0077/ $\$ 1,00+\$ .15$ per page. Paper No. 25834. 
ferometry (VLBI) antenna at the same location. It also incorporates two stations FLNR C1 (near Gainesville) and MCDAVID RM A (north of Pensacola) belonging to the NGS GPS eastern strain network (ESN) that were previously connected through GPS observations to a set of fiducial fixed and mobile VLBI sites.

As a secondary goal, not discussed in this paper, about 145 azimuth marks were positioned during the same observing campaign employing single frequency receivers and using faster kinematic procedures (Taylor 1989). They followed first-order geometric positioning standards relative to the primary HPN. In addition, GPS vertical-control connections were also established, in order to achieve tide gauge ties as requested by the FDNR.

\section{Data Collection and Processing}

The field data of the Florida HPN contain a total of 156 sessions, involving from two (43 sessions) to five (17 sessions) TI-4100 four-channel, dual-frequency receivers equipped with GESAR (geodetic satellite receiver) data-acquisition software. Due to unexpected thunderstorms, the data collected at some sites were considered to be of low quality (if the overall requirement of $1 \mathrm{ppm}$ was to be met) and single vector reobservations (25 sessions of the 43 already mentioned) were required. The project occupied 252 stations; 71 NAD 83 (1986) points (62\% first-order), 64 bench marks, 33 of which were both horizontal and vertical control points. Of all these stations 173 are considered B-order (Standards 1988) and were occupied at least twice. A total of 412 independent GPS vectors were determined in the

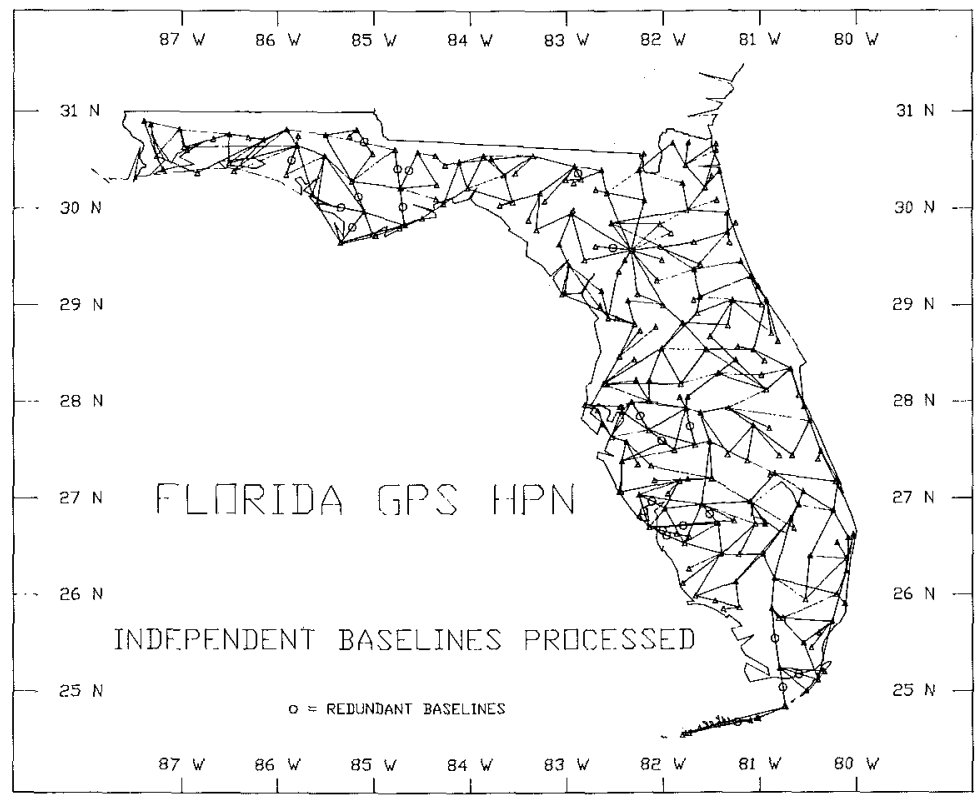

FIG. 1. Independent Baselines Reduced in Florida High-Precision Network 
data reduction (see Fig. 1). Baselines observed more than once during different sessions are represented with a circle in the figure. Spur lines are mainly connections to vertical bench marks.

Processing, in the multivector (solving for tropospheric biases and cross correlations between vector components) mode, was implemented on DELL (System 310) and EVEREX 386/20 Personal Computers using the OMNI software package version 1.0 developed at NGS (Mader et al. 1990). The selected measurement interval for the main project was $30 \mathrm{~s}$, and reobservations were collected and processed using a 15-s interval. In both cases a minimum elevation angle of $20^{\circ}$ was selected as the cut-off angle for all carrier phase observables. In order to guarantee the best satellite geometry, observation spans of 5-6 hours were scheduled for each session. This time window assured at least six different satellites above the horizon during the complete observing period, usually a minimum requirement when high accuracy is needed. Specifically, satellites with pseudo-random-noise (PRN) code numbers $3,6,8,9,11,12$, and 13 were observed. However, due to the severe weather conditions (in one occasion lightning hit a GPS antenna) and other minor hardware failures, only 3 hours of observations were available at four stations (FLGPS 65, FLGPS 2, MISSILE and FLGPS 4). Furthermore, as Fig. 2 depicts, the satellite geometry was not optimum. Notice from the satellite sky plots that no satellite tracks overlap the second quadrant. With the advent of a full GPS constellation and new instruments that can track all satellites in view, the satellite geometry should be improved

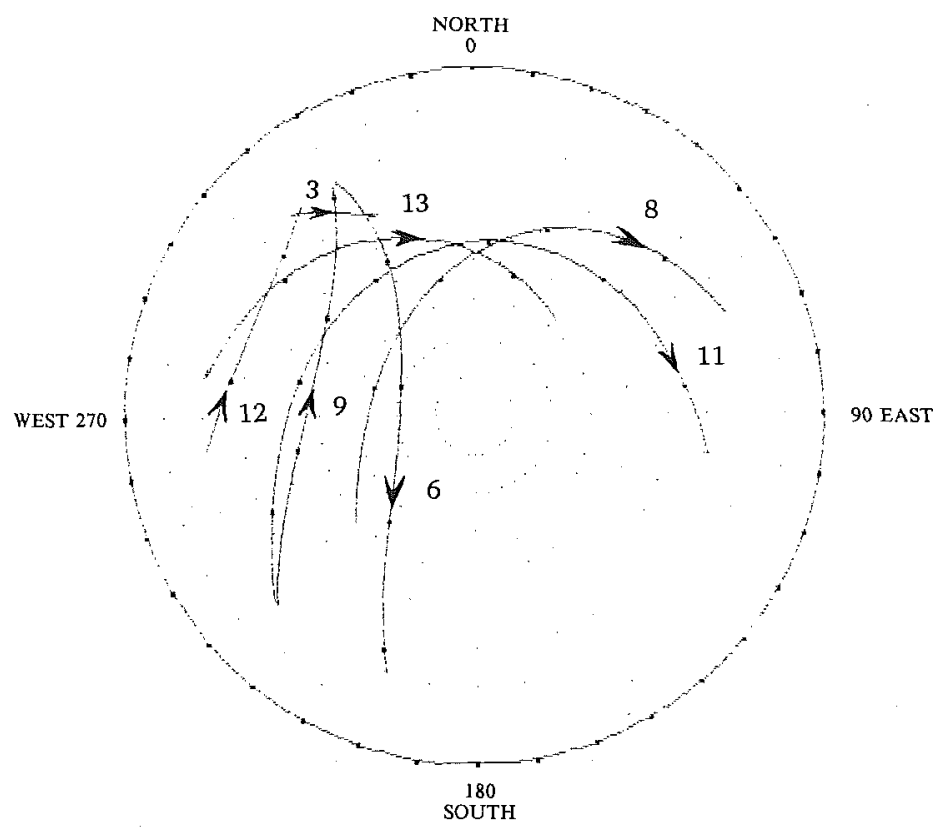

FIG. 2. Satellite Sky Plot; Latitude $=28^{\circ} 0^{\prime} 0^{\prime \prime} \mathrm{N}$; Longitude $=81^{\circ} 30^{\prime} 0^{\prime \prime} \mathrm{W}$; Date April 22, 1989 (Day 112); Start Time 0 hours 0 min, 0 s UTC, Final Time 6 hours, $30 \mathrm{~min}, 0 \mathrm{~s}$ UTC 
and the observation time span per session can possibly be reduced considerably.

Precise postfitted ephemerides generated at the Naval Surface Warfare Center (NSWC) and referred to the World Geodetic System of 1984 (WGS 84) were used in all reductions. From GPS week 485 (day of year 113) on, by a decision made at NSWC, the state vector for satellite PRN 8 was not included in the precise ephemerides transmitted to NGS. Although the frequency standard of satellite 8 was erratic in the past, it did not cause any apparent problems during the observing period of the Florida project. This satellite was finally deactivated on October 14, 1989. Thus from April 23, 1989 , onward the precise ephemerides of only six satellites were available to process the data. This fact may have degenerated the mathematical model geometry for each individual session after that date. Although the full impact on the solutions of the loss of this particular satellite is difficult to assess without a careful independent analysis, it is plausible that significant systematic effects in the vertical component may have been introduced if the observations from PRN 11 [the only satellite covering the first quadrant (see Fig. 2)] were affected by local weather fronts or other unpredictable data problems.

After all nonautomatically corrected data outliers and cycle slips (on frequencies L1 and L2) were manually cleaned (relying as reference on OMNI's postfitted residual plots), final solutions were determined using the L3 option, which minimizes the effect of ionospheric refraction. Ambiguity biases were fixed whenever possible. There was a total of 119 sessions $(76 \%)$ where it was possible to fix all integers. The rest were only partially fixed solutions.

\section{REsults}

A minimally constrained network adjustment using NGS's three-dimensional least-squares adjustment program (LAP) was performed. Station TIMER was held fixed to coordinates referred to a VLBI-defined frame previously established by independent solutions using observations to radiosources from fixed VLBI instruments around the world. Due to large observational residuals, primarily caused by unstable atmospheric conditions during electric storms, it was necessary to reject $15(3.6 \%)$ GPS vectors. The sole criterion applied to exclude these observations was their large residuals (the ones generally exceeding a preselected tolerance of $5 \mathrm{~cm}$ in the east and/or north components or $20 \mathrm{~cm}$ in the height component). These observations were also rejected earlier in a preliminary LAP solution using vectors where the integers were not held fixed, clearly pointing out deficiencies in the original raw data.

The adjustment a posteriori standard deviation of unit weight was 4.87 . Although this value may appear large, it is very much in agreement with the order of magnitude expected for such GPS projects. It is a well-known fact [e.g., Craymer et al. (1990)] that conventional routines for predicting a priori weight matrices for the GPS observables (double differences carrier beat phase) give overoptimistic estimates for the standard errors of the reduced vector components. It should be stressed here that the resultant covariance matrix of the vector components was not scaled before it was used in the network adjustment. Thus it appears that in this case, the OMNI- 
generated standard deviations ("formal" errors) of the vector components are optimistic on the average by a factor of five.

The project specifications, in accordance with geometric geodetic accuracy standards provisionally accepted by the Federal Geodetic Control Committee (Geometric 1988), required B-order horizontal-control accuracy $(8 \mathrm{~mm}+$ $1: 1,000,000$ or $8 \mathrm{~mm}+1 \mathrm{ppm})$. The vertical control, based on ellipsoidal heights, was targeted to a precision of $5 \mathrm{~cm}$.

Project requirements were amply met for the horizontal control. Initially, a sample of 76 loop misclosures (from the many possible) was computed using only independent vectors. In order to confront the most stringent situation, priority was given to triangular misclosures. Two-dimensional relative errors from all these loops were always better than $1 \mathrm{ppm}$ and only four loops fell between $1: 1,000,000(1 \mathrm{ppm})$ and $1: 2,000,000(0.5 \mathrm{ppm})$. The maximum 2-D relative error detected in these four loops was $0.72 \mathrm{ppm}$ and this particular circuit was identified as having observations affected by thunderstorms. With respect to the 3-D loop misclosures, 24 of them (due to the height component errors) did not meet the $1 \mathrm{ppm}$, but only one of these was above $2 \mathrm{ppm}$ (exactly $2.21 \mathrm{ppm}$ ). By rejecting the 15 inconsistent observations that were mentioned previously, as expected, most of these loops were later improved. These results once more emphasize the well-known, but not yet satisfactorily explained, weakness of the vertical components as derived by GPS methods when compared to the horizontal results. It is generally assumed that the precision of the vertical component will be worse than the horizontal components by a factor of two to five. This factor may be close to one only when short vectors are involved, and common errors (i.e., orbits, ionospheric and tropospheric refraction, etc.) at both stations are minimized by the differencing algorithm creating the observables. [An empirical proof is given by Hajela (1990)].

As a further measure of internal consistency of the network, the precision of all independently observed repeated vectors was also studied. In this project there were only 23 vectors (averaging $41 \mathrm{~km}$ in length) that were repeated during different dates and sessions, an average of 15 days apart. The repeatability of their length in all cases was much better than $1 \mathrm{ppm}$, the largest relative error (between stations HAVO and PUNT) being $0.66 \mathrm{ppm}$.

These two types of computations (loop misclosures and vector length repeatability) are only measures of the network internal consistency. In order to obtain some idea of accuracy, an external and independent reference must be found. It was assumed that the published NAD 83 (1986) positions, because of their intrinsic qualities [the result of a simultaneous adjustment that included Doppler coordinates and all archived classical observations (Schwarz and Wade 1990)], are not overall at the same level of accuracy as the GPS determined values. The corroboration of this assertion will become evident later when some comparisons are introduced.

To date, the only available standard of accuracy equal to or better than the GPS results rests on VLBI and/or satellite laser ranging (SLR) techniques, which are based on more refined, albeit complicated, instrumentation and methods. As already mentioned, one station (TIMER) forms part of the HPN but it also belongs to the NAD 83 (1986) and it is connected to the VLBI-fixed global network. Thus, its coordinates are rigorously known in this VLBI/SLR defined reference frame, specifieally the one called National Earth Orientation Service 1990 (NEOS 1990) ("NEOS" 1990). Eventually 
TABLE 1. Comparison of NEOS 1990 and GPS-Derived Geodetic Coordinates at Stations FLNR C1 and MCDAVID RN A; Differences Are Given in Sense GPS Minus NEOS 1990

\begin{tabular}{|c|c|c|c|c|c|c|}
\hline \multirow[b]{2}{*}{$\begin{array}{c}\text { Stations } \\
\text { (1) }\end{array}$} & \multirow{2}{*}{$\begin{array}{c}\Delta \phi \\
(\mathrm{cm}) \\
(2)\end{array}$} & \multirow{2}{*}{$\begin{array}{c}\Delta \lambda \\
(\mathrm{cm}) \\
(3)\end{array}$} & \multirow{2}{*}{$\begin{array}{c}\Delta h \\
(\mathrm{~cm}) \\
(4)\end{array}$} & \multirow{2}{*}{$\begin{array}{c}\text { Distance to } \\
\text { TIMER (m) } \\
(5)\end{array}$} & \multicolumn{2}{|c|}{ Relative Accuracy } \\
\hline & & & & & $\begin{array}{l}2-D \\
(6)\end{array}$ & $\begin{array}{c}3-D \\
(7)\end{array}$ \\
\hline FLNR & 0.72 & 10.20 & 0.60 & 479 & $2.13 \times 10^{-7}$ & $2.14 \times 10^{-7}$ \\
\hline MCDAVID RN A & 21.00 & 13.80 & -15.50 & 896,341 & $2.80 \times 10^{-7}$ & $2.31 \times 10^{-7}$ \\
\hline
\end{tabular}

(McCarthy 1989), the International Earth Rotation Service (IERS) is introducing the IERS Terrestrial Reference Frame (ITRF) based on a combination of various solutions (e.g., NEOS 1990) as the standard geocentric earthfixed frame to be used in all geodetic work. Because TIMER is part of the global network of points defining the ITRF and its coordinates are presently accurately known it was selected as the fixed station in the Florida minimally constrained least-squares network adjustment. Recall that the Florida GPS HPN also includes stations FLNR C1 and MCDAVID RN A, the coordinates of which are also known in the NEOS 1990 frame and were derived from an unrelated adjustment of the GPS eastern strain network. In this adjustment the coordinates of some GPS stations (collocated with VLBI points) of the ESN were constrained to the NEOS 1990 values. When the coordinates of FLNR C1 and and MCDAVID RN A resulting from the minimally constrained network adjustment (fixing TIMER) using all GPS vectors were checked against their known values in the NEOS 1990 frame, the outcome was remarkable. Table 1 shows the computed differences. Notice that when the GPS results are compared to an independent and reliable source of accuracy, the two- and three-dimensional relative errors are always well inside the 1-ppm limits (about $0.2 \times 10^{-6}$ ); discrepancies are primarily due to the noncoincidence of the WGS 84 (materialized through GPS observations) and the VLBI/SLR NEOS 1990 frames.

The important consequence of this type of comparison is that, if high standards of accuracy are required for future GPS high-precision networks already in the observational phase or firmly projected for various states (Strange 1990), more mobile VLBI fiducial points (or GPS stations with coordinates known in the ITRF frame) must be established. This is now a validation procedure at NGS for providing independent and accurate external standards with which to compare and judge the final GPS results. As an added byproduct, this GPS A-order "supernetwork" will create the necessary framework for connecting in a common reference frame all the GPS high-precision B-order networks for each individual state, thus avoiding possible minor inconsistencies at the bordering regions. The problem is more complicated along the borders of adjacent states with and without HPN. Although several alternatives are available, the policies of NGS on this respect have recently been decided and published (Bodnar 1990b).

The initial vertical control requirement of $5-\mathrm{cm}$ precision in ellipsoidal height was not totally achieved in this project, probably because of the following three reasons:

1. The special local weather conditions encountered in southern Florida (primarily thunderstorms and high humidity). 
2. The erratic behavior of the ionosphere during 1989 (a peak in the 11-year sunspot cycle).

3. The less-than-optimum satellite geometry.

Points 1 and 2 are problems directly affecting heights that require more sophisticated refraction modeling (e.g., tropospheric and ionospheric propagation delays) than the ones currently implemented in most GPS-reduction software packages. This is especially critical when rapidly changing atmospheric conditions are approximated by using averaged (three epochs) surface measurements of pressure, temperature, and humidity.

Also, comparisons with an external physical standard (leveled orthometric heights) were examined. However, in this approach it is mandatory to have geoidal heights or undulations. These were predicted using the recently released OSU89B $360 \times 360$ potential model (Rapp and Pavlis 1990). Although the estimated absolute accuracy of this type of model is generally quoted (for nonmountainous regions in the United States) on the order of 0.5-1 $\mathrm{m}$, nevertheless, this figure may be slightly optimistic in Florida. When the GPS ellipsoidal heights converted to orthometric heights using the OSU89B model were compared to the NGVD 29 values some discrepancies, possibly due to a systematic slope of the modeled geoid, were detected. This subject is expanded later in the text.

Independent of these modeled geoidal heights was the geometric comparison with respect to the NEOS 1990 frame. As Table 1 showed, the differences in ellipsoidal heights at FLNR C1 and MCDAVID RN A between the minimally constrained LAP solution (with TIMER held fixed to the NEOS 1990 values) were respectively $0.6 \mathrm{~cm}$ and $-15.5 \mathrm{~cm}$. When the total distance between the points is considered, the agreement is well within the maximum closure expected in first-order class II leveling (i.e., $5 \mathrm{~mm}$ $\sqrt{\mathrm{E}}$, where $E=$ length in $\mathrm{km}$ ) (Standards 1984). One can weigh these results against the substantial expense incurred when lines of this length are leveled by conventional methods.

It is important to emphasize that these high-precision geodetic networks are observed in a very restrictive temporal domain. That is to say, no extra time is generally available for redundant multisession vector observations. Thus, due to this infrequent repeatability, the results may lack the standards of those published by other GPS investigators (e.g., monitoring deformations) who base their analysis and conclusions on many repeated measurements of the same vectors, usually collected under many different sets of circumstances. This contrasts dramatically with situations such as in this project. The HPN is almost totally dependent on only one observation per vector, many times obtained in less-than-ideal conditions. Unfortunately, this is the case in most GPS geodetic and surveying applications where the logistics of cost-effective performance is an unavoidable priority. Florida was no exception. In the present work, the height results are clearly affected by this crucial time restriction. That is, the lack of redundancy (although every Border station was occupied at least twice) combined with unpredictable weather conditions resulted in poorer than normally expected height determinations. Therefore, the ellipsoidal (geodetic) height results did not reach the level of precision that other GPS researchers have previously experienced under almost "laboratory environments" and openly announced. Here we have confronted a real world subjected to practical limitations. Nevertheless, GPS is 
the best possible alternative to geodetic and surveying measurements, and its present level of accuracy easily supersedes any requirement for current mapping or GIS/LIS needs. Moreover, with better modeling of refraction, improved satellite configuration, and precise orbits referred to a well-determined terrestrial reference frame, the limit of GPS accuracies is not yet in sight.

Figs. 3 and 4 depict the Florida HPN adjustment residuals projected on the planes of the geodetic horizon (east versus north) and geodetic meridian (east versus height $=$ up). The plots in the figures encompass every observation residual as obtained from the least-squares network adjustment. Notice, for example, that the horizontal displacements do not exceed, respectively, $\pm 1.5 \mathrm{~cm}$ and $\pm 2.5 \mathrm{~cm}$ in latitude and longitude. The larger error in longitude is properly explained by the difficulty of perfectly modeling the rotation of the earth and the peculiar satellite coverage.

Furthermore, some of these residuals are referred to vectors with the same remote (in this context repeated) station, which is the end point of several independent vectors measured during different sessions. The practical effect of the adjustment is to "move" the initial values of the station coordinates by an amount equal to its residual. Then, if more than one residual applies to the same point, this will be "displaced" by an amount equal to its "average residual" after the adjustment. In other words, as theory predicts, the final coordinates of the station will be the "best" in a least-squares sense (i.e., resulting from minimizing the sum of the squares of all residuals), in essence selecting the "center of gravity" of all residuals involved as a solution for each individual station. This is why it is so critical for any statistical analysis to have repeated observations.

To visualize the significance of repeatability, Figs. 5 and 6 represent the final individual residuals (a single value per component for each station) after the appropriate averages were taken. As anticipated, the dispersion is greatly reduced. The relevance of repeatability is graphically accentuated when Figs. 3 and 4 are compared with 5 and 6 . The residual plots, in principle, display the two-dimensional view of the root-mean-square (RMS) of bivariate random variables, giving an intuitive understanding of the spatial precision of the final coordinates.

Recall that the initial standard deviations of the reduced GPS components are always optimistic (systematic biases due to orbital, atmospheric, and multipath errors are unmodeled). Therefore, the final values of the standard deviations (the so-called formal errors) of the adjusted parameters (coordinates) are too small. Hence, from Figs. 3 and 5, and completely independent of formal statistics, it is possible to deduce that the curvilinear geodetic coordinates (latitude and longitude) were determined to better than $\pm 1 \mathrm{~cm}$. The maximum RMS of these estimates about their mean is only $0.59 \mathrm{~cm}$. These results are nothing less than outstanding for a geodetic network of this size. Considering that the average length of the baselines is $45 \mathrm{~km}$, it represents that in the worst possible case (when the error may be $1 \mathrm{~cm}$ ) the achieved horizontal relative accuracy is about $2 \times 10^{-7}$ or $0.2 \mathrm{ppm}$. Accordingly, with regard to the horizontal positions, it can be said that they are more than a 20 -fold improvement over the most optimistic estimates for the NAD 83 (1986) datum. These results would be unachievable economically without the application of GPS technology. When geodetic networks of the extension and peculiar characteristics of this project are considered, 


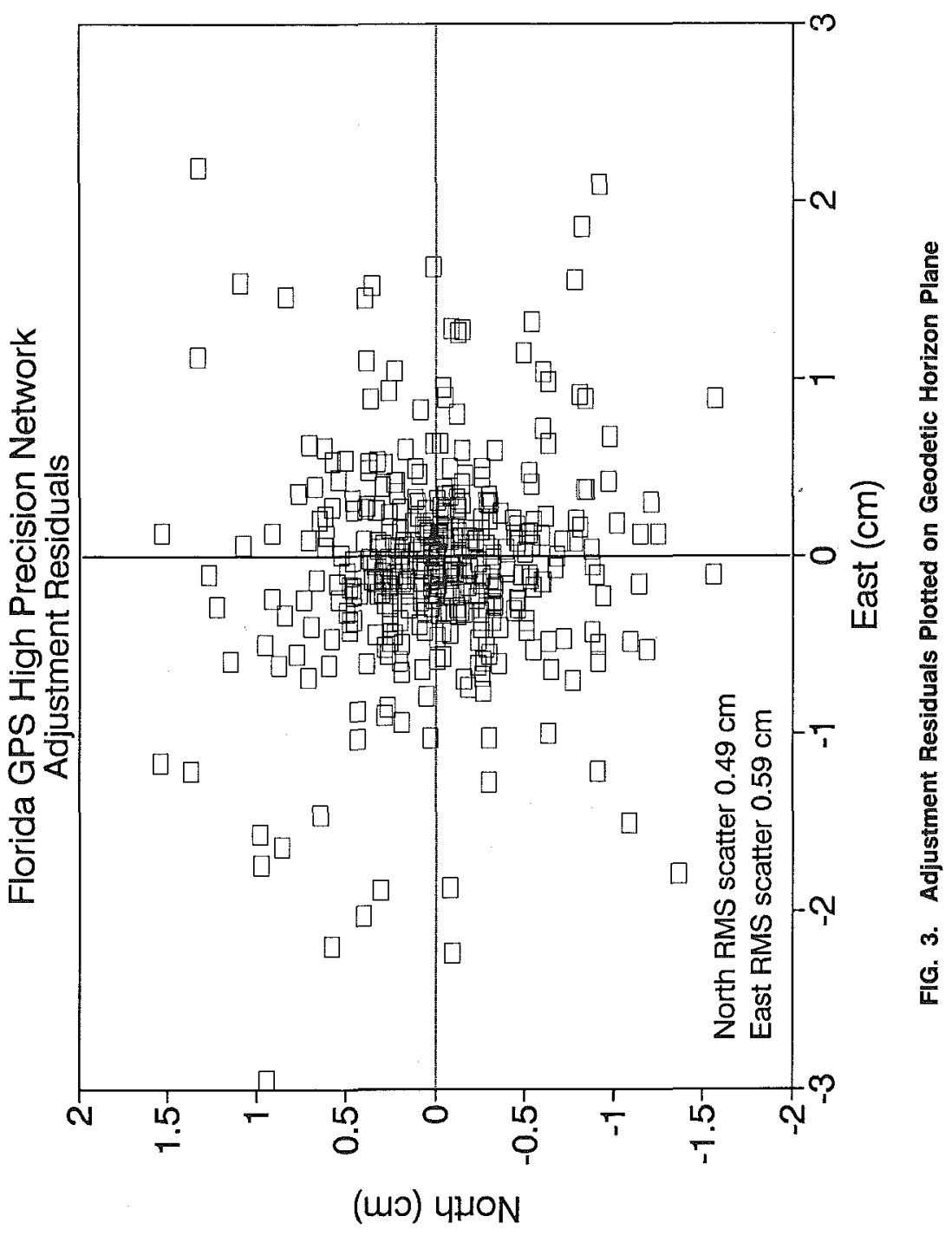




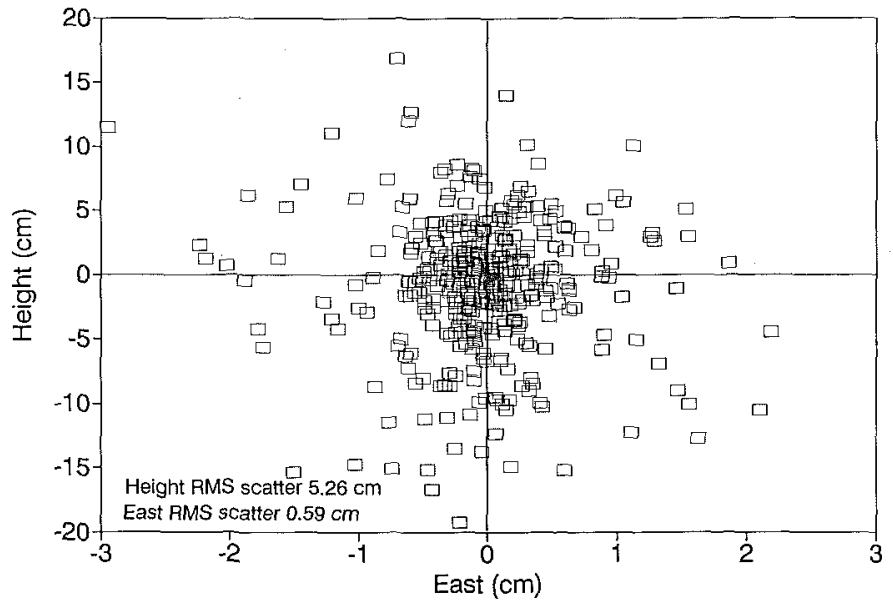

FIG. 4. Adjustment Residuals Plotted on Geodetic Meridian Plane

GPS is the only sophisticated and effective procedure capable of doing the job at this level of relative accuracy and cost.

On the other hand, Fig. 6 also shows that the height component is within the range of $\pm 10 \mathrm{~cm}$ except for two points which nevertheless are very well determined (to a few millimeters) in longitude and latitude. However, notice that most of the geodetic height residuals are clustered around the $\pm 5 \mathrm{~cm}$ limits, thus determined to this level of precision.

Consequently, at least in this Florida project and due probably to the ex-

Florida High Precision Network

Adjustment (Station Average) Residuals

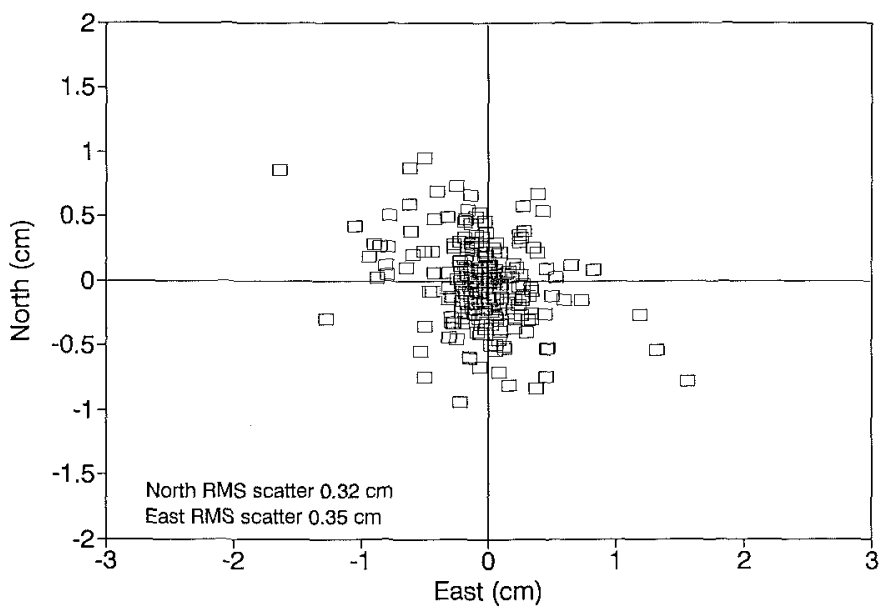

FIG. 5. Residuals (Station-Averaged) Plotted on Geodetic Horizon Plane 


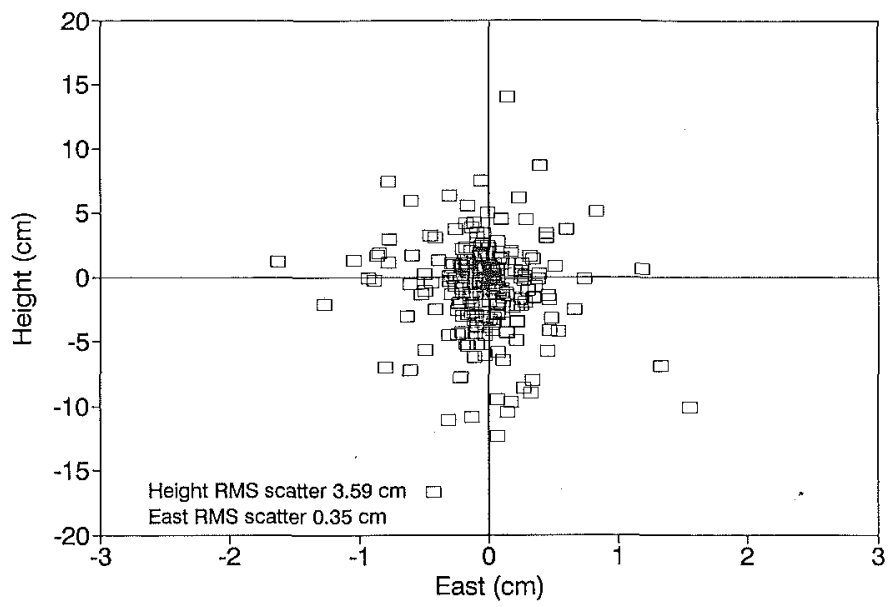

\section{FIG. 6. Residuals (Station-Averaged) Plotted on Geodetic Meridian Plane}

treme atmospheric conditions already mentioned, it was impossible to obtain the precision of all ellipsoidal heights well inside the $5-\mathrm{cm}$ requirement. The worst RMS scatter is $5.26 \mathrm{~cm}$ (see Fig. 4). The magnitude of the vertical and horizontal errors are not necessarily correlated, clearly implying that they depend on independent error sources. Longitude and latitude determinations are primarily affected by the rotation of the earth and satellite geometry; the height errors by how uncertainties in the atmospheric modeling influence the incoming signal from each individual satellite.

Finally, in order to see the distribution of residuals with respect to mag-

Florida GPS High Precision Network Residuals (East \& North) Histograms

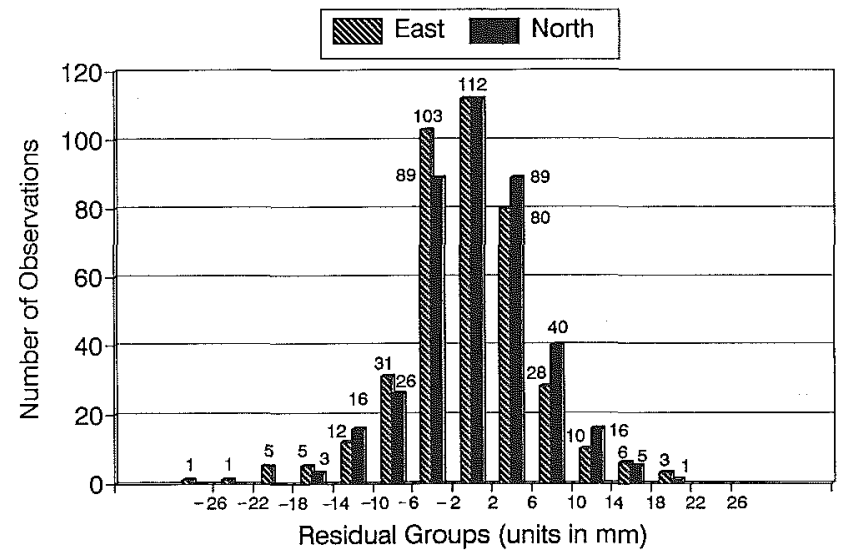

FIG. 7. Histograms of Residuals along East and North Components 


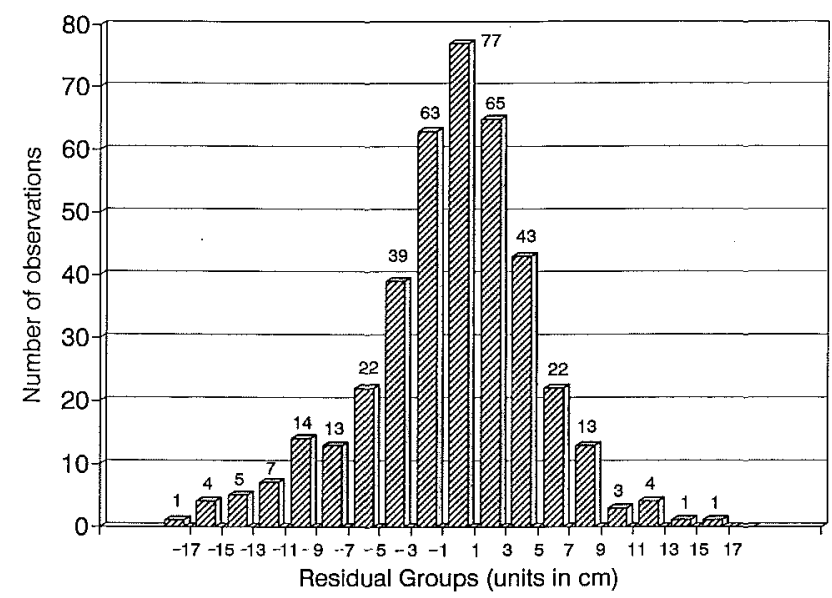

FIG. 8. Histogram of Residuals on Vertical (Height) Component

nitude, Figs. 7 and 8 show the histograms of the residuals (corresponding to Figs. 3 and 4) along the east, north, and height components.

\section{CCMPARISONS OF NAD 83 (1986) In FLoRIDA With GPS AND VLBI}

To assess the quality of NAD 83 (1986) in Florida, several comparisons were inade. Station TIMER was fixed to the published NAD 83 (1986) coordinates and a minimally constrained network adjustment was performed using ali GPS vectors. Fig. 9 graphically depicts the results. The GPS coordinates were obtained through a minimally constrained network solution using all GPS vectors and station TIMER fixed to the published NAD 83 (1986) values. A perusal of the magnitude of the plotted differences indicates that the principal goal of the NAD 83 (1986) readjustment has been fully accomplished.

When the original curvilinear geodetic coordinates in the NAD 27 datum were transformed to Cartesian coordinates and compared (after shifts, rotations, and scale were considered) to the Doppler solution (in the NWL 9D quasi-geocentric system), residual displacements were encountered (as large as $2.5 \mathrm{~m}$ in latitude and $2.0 \mathrm{~m}$ in longitude in Florida). In order to correct for these significant but random distortions, implicit in the NAD 27, plus the incorporation of geocentricity and the change to the ellipsoid of the Geodetic Reference System of 1980 (GRS 80), a readjustment using all classical observations was performed where the coordinates of about 590 Doppler points (plus 112 VLBI baseline components and five GPS survey ties in Alaska) were constrained to a certain level of precision. The resultant NAD 83 (1986) eliminated, in an aver :ge sense, these large regional deformations; nevertheless, smaller and very ". calized distortions may still be present (and undetected) at certain locations uround the country [see Snay (1990)].

The NAD 83 (1986) results in Florida are good judging by the differences 


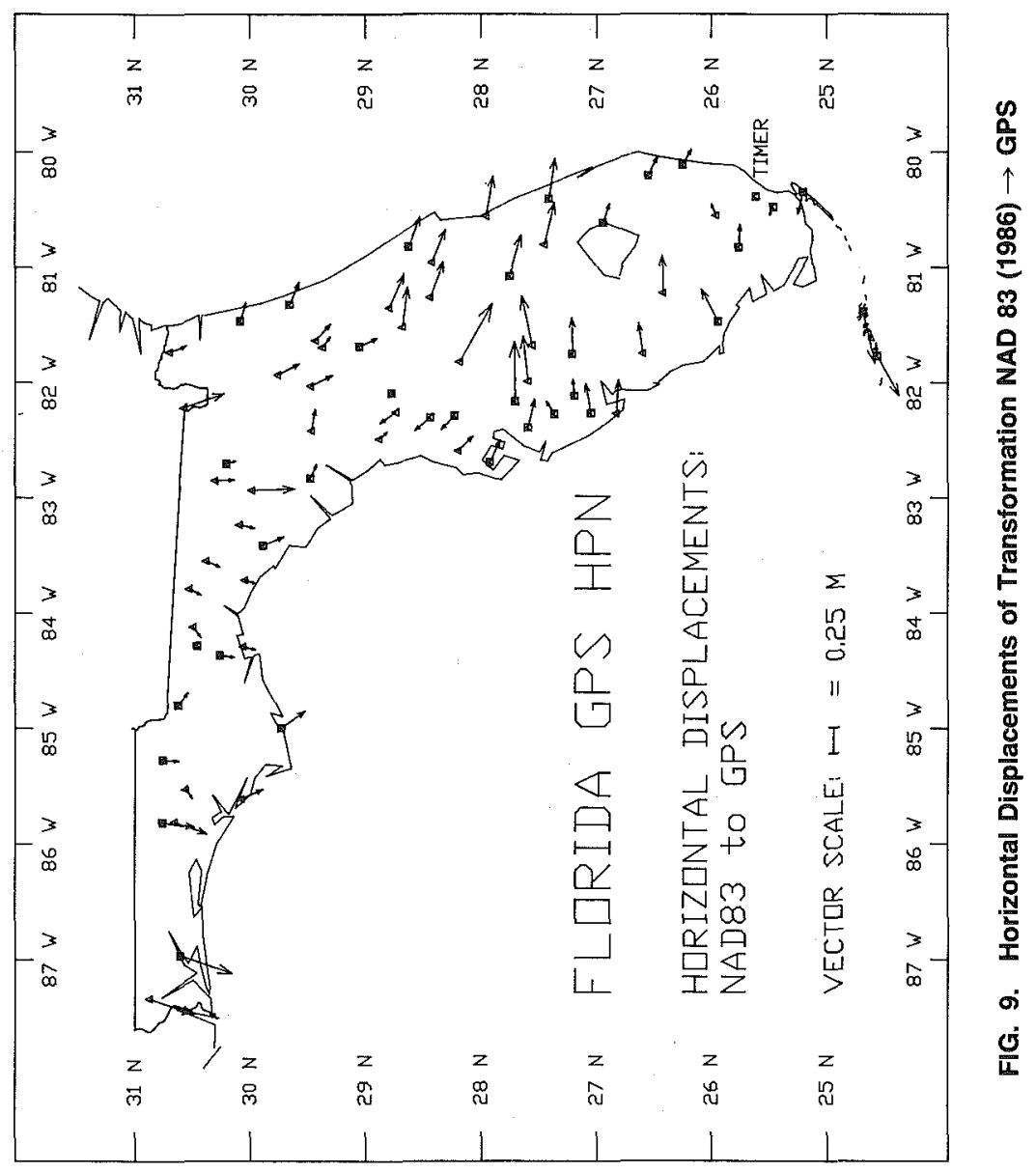


between the recently determined GPS coordinates and the published NAD 83 (1986) values. The maximum displacement reaches a magnitude slightly larger than $0.5 \mathrm{~m}$ at station POLK RESET, which is approximately located in the center of the state. Because the internal consistency of the GPS results, as already discussed, was only on the order of $\pm 1 \mathrm{~cm}$, and even the absolute comparisons with the NEOS 1990 coordinates around the $\pm 15 \mathrm{~cm}$ range, the plotted displacements in Fig. 9 can only be attributed to remaining random errors in the NAD 83 (1986). In particular, the GPS residuals attached to station POLK RESET were only: east $=0.08 \mathrm{~cm}$ and north $=-0.27 \mathrm{~cm}$. It is speculated that, because this is a new site that replaced an old mark, the possibility of inadvertently mixing in the NAD 83 (1986) readjustment observations referred to the two stations as plausible. As a consequence, due to the ties with nearby points, these errors may have been propagated to other marks in the surrounding area. An investigation is under way to explain the problem properly, although this will be totally irrelevant once the new GPS coordinates at the points are adopted as reference. In any case, it is clear from the figure that in Florida when the NAD 83 (1986) coordinates are compared to the new GPS HPN, the relative errors in the adopted horizontal datum are (with respect to TIMER) better than 1:400,000. This amply surpasses the projected target at the time the readjustment was planned, and exceeds the original accepted estimates of 1:200,000 (Ethridge 1989). The reader is undoubtedly aware that the vector representation of Fig. 9 is purely relative and depends on the choice of the constrained station. That is why it is so important to fix a well-defined point (e.g., TIMER), in the minimally constrained adjustment. In the worst possible scenario, assuming that POLK REST [a probable outlier in the NAD 83 (1986)] is selected as the constrained station, maximum relative errors of about $1: 100,000$ with respect to some nearby points are obtained. This clearly indicates that localized distortions in some states may be still far worse than the precision achievable with modern GPS techniques.

Finally, Fig. 10 depicts the displacement vectors between coordinates referred to the horizontal NAD 83 (1986) and their counterpart in the NEOS 1990 frame. In this case TIMER was held fixed to its NEOS values and the results of the network adjustment using all GPS vectors, compared to the published NAD 83 (1986) values. The figure shows systematic differences between the two sets of coordinates, but again, the quality of the agreement is also surprising. It can be concluded, judging by the magnitude of the displacements, that the geocentricity of NAD 83 (1986) is a practical reality. To understand better the relationship between the two frames, a seven-parameter similarity transformation was performed. It should be kept in mind that NAD 83 (1986) is a horizontal datum, consequently, the geodetic (ellipsoidal) heights were not estimated in the readjustment and they are only approximately known. Actually, geodetic heights were determined using the best available information at the time and held fixed in the readjustment except where Doppler, VLBI, or GPS data were observed (Snay 1989). Hence, although the GPS solution computes the geodetic height of each station in the WGS 84 datum, they were presumed zero when the transformation parameters were sought. Thus (as it is required), we assumed both sets of points on the surface of the GRS 80 ellipsoid. This way the possibility of introducing scale errors due to differences in assumed NAD 83 (1986) and GPS-obtained ellipsoidal heights is avoided. 


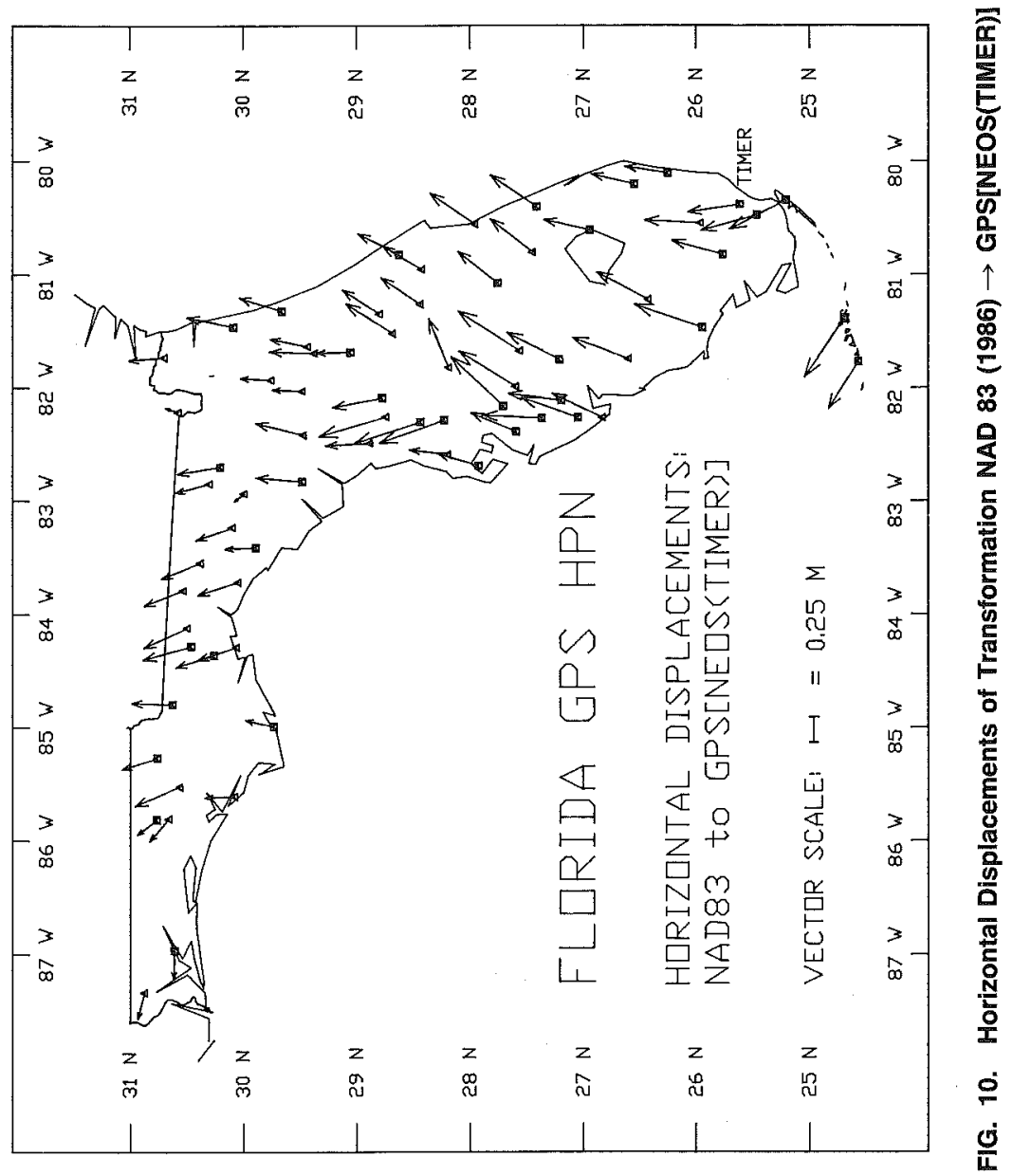


TABLE 2. Parameters of Similarity Transformation NAD 83 (1986) (Florida) $\rightarrow$ GPS[NEOS(TIMER)]

\begin{tabular}{c|c|c|c|c|c|c}
\hline \hline $\begin{array}{c}\Delta \mathbf{x}(\mathrm{m}) \\
(1)\end{array}$ & $\begin{array}{c}\Delta \mathbf{y}(\mathrm{m}) \\
(2)\end{array}$ & $\begin{array}{c}\Delta \mathbf{z}(\mathrm{m}) \\
(3)\end{array}$ & $\begin{array}{c}\delta \epsilon(\operatorname{arcs}) \\
(4)\end{array}$ & $\begin{array}{c}\delta \psi(\operatorname{arcs}) \\
(5)\end{array}$ & $\begin{array}{c}\delta \omega(\operatorname{arcs}) \\
(6)\end{array}$ & $\begin{array}{c}\delta s(\mathrm{ppm}) \\
(7)\end{array}$ \\
\hline 0.11 & 0.13 & 0.44 & -0.007 & 0.049 & -0.027 & -0.017 \\
\pm 0.05 & \pm 0.36 & \pm 0.19 & \pm 0.002 & \pm 0.011 & \pm 0.006 & \pm 0.002 \\
\hline \hline
\end{tabular}

First, a four-parameter (three shifts and one scale factor) similarity transformation was determined. Then, the values of the computed translations were constrained and the scale and three rotations estimated. Table 2 (for an explanation of the notation see Appendix I) shows the least-squares solution for the seven parameters in the sense NAD 83 (1986) $\rightarrow$ GPS [NEOS (TIMER)]. No observations were rejected and the a posteriori standard deviation of unit weight was 1.7 .

These results once more qualify the NAD $83(1986)$ as a successful scientific undertaking. Small differences in shifts, scale, and rotations are present, but not at a discouraging level. By properly weighing classical observations (constrained by Doppler positions), added to the astute application and development of modern computer hardware and software [e.g., Helmert blocking (Schwarz and Wade 1990)], a historical geodetic first was achieved.

The final comparisons clearly show the influence of the Doppler constraints upon NAD 83 (1986). A residual bias of about $0.4 \mathrm{~m}$ is still present in the z-shift, possibly indicating that the adopted value of $4.5 \mathrm{~m}$ for the transformation NWL 9D $\rightarrow$ WGS 84 (Soler and Hothem 1989) and implicit in the constraints of NAD 83 (1986), should have been closer to $5 \mathrm{~m}$. Incidentally, Table 2 applies only to Florida and should not be generalized for the entire country. Summarizing, it can be said that at least in the state of Florida, the projected quality of the NAD 83 (1986) was not only achieved, but even surpassed without reservations.

\section{Comparison of Orthometric Heights Derived from GPS and Global Geoidal Model With NGVD 29}

Finally, a comparison between orthometric heights determined using GPS and a geoidal model (OSU89B) with the adjusted leveled heights of NGVD 29 was completed. The analysis showed that current global geoidal models may be too crude to be used with the GPS results if accurate orthometric heights are desired. The results of this investigation also indicate a conceivable slope in the modeled global geoid in Florida. Some outliers of about 1 $\mathrm{dm}$, obviously few and random (see Fig. 6), are present in the GPS-determined ellipsoidal heights; however, from Fig. 11 [values are given in the sense NGVD $29 \rightarrow$ GPS (OSU89B)] it is clear that a systematic trend is implicit in the plotted differences. Other solutions using GPS-derived ellipsoidal heights from different minimally constrained network adjustments gave an equivalent systematic trend, supporting the hypothesis that the slopes of the "true" and modeled geoid in Florida do not coincide. In retrospect, the selection of TIMER was the appropriate one, considering that the NGVD 29 leveled orthometric height of this bench mark is $4.17 \mathrm{~m}$ and the computed value using the OSU89B model and NEOS 1990 ellipsoidal height $4.31 \mathrm{~m}$. 


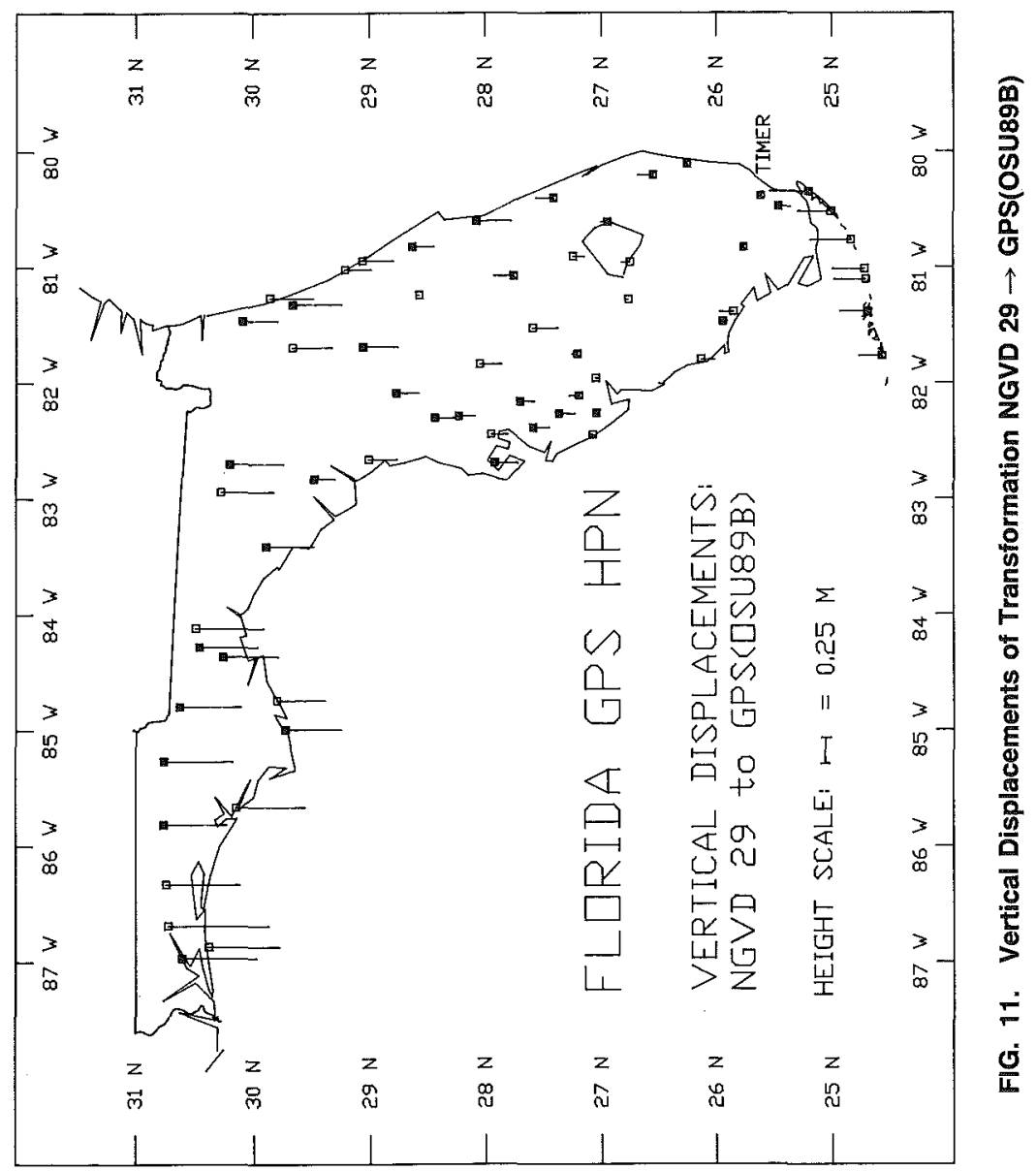


Thus, at worst, the vertical displacements in Fig. 11 may have an absolute bias of only $14 \mathrm{~cm}$.

All indications point to the possibility that the true geoid in Florida is smoother than the model appears to indicate. We need to realize that the geoidal model may have an absolute accuracy of only about $1 \mathrm{~m}$. However, as seen from the figure, this should not affect greatly the determination of relative geoidal heights between points that are not too far apart [3-4 ppm of the distance for the OSU89B (Rapp and Pavlis 1990)]. Therefore, the absolute accuracy of the geoid model OSU89B is still an open question that will require more comparisons to be answered conclusively. Other analyses in Florida using orthometric heights from different leveling adjustments are under way at NGS, and future research may explain and/or correct the obtained differences.

Currently, there are only two alternatives for accurate vertical work: (1) To depend on leveled bench marks as a source of orthometric height information; or (2) to use GPS-determined ellipsoidal heights directly without attempting to convert them to orthometric heights.

To improve our GPS determination of orthometric heights the necessity of generating a continental national geoid, initially with absolute accuracies at the decimeter level, is an important priority. NGS is actively reviewing the feasibility of such an enterprise and its possible benefits to the geodetic and surveying community. It is envisioned that the task of determining such an accurate geoid may be completed desirably by early 1991 with further upgrades planned for the future (Bodnar 1990a).

\section{Conclusions}

In conclusion, and considering the quality of the data, it is encouraging to state that the primary objectives of NGS and the Florida project were surpassed with respect to the horizontal control. In relation to the vertical control, more studies should be initiated to understand properly the full dependency of GPS-determined ellipsoidal heights with signal propagation. It is obvious that more improved and complex mathematical models capable of rigorously correcting for tropospheric and ionospheric refraction are required when the atmospheric conditions deviate from the standard. Meanwhile, the geodetic engineer must rely on leveled observations for accurate (first-order) orthometric height determination, until the uncertainties affecting the computation of heights using GPS under atypical circumstances and the systematic errors contributed by global geoidal models are understood. Until these goals are realized, and exclusively based on the results of this experience, it is recommended that when accurate vertical control is desired, GPS observations in subtropical areas be limited to the dry season to minimize errors in the vertical component.

Finally, the results indicate that in the state of Florida the relative accuracy of NAD 83 (1986) can be established between 5 and 2 ppm, surpassing the most optimistic assumptions of any previously projected estimates, although inferior to the capabilities of modern GPS techniques and methods.

\section{ACKNOWLEDGMENTS}

Many people from NGS contributed to the success of this project and were 
directly involved in many of the different facets that a complex endeavor of this kind requires. In particular we would like to thank Field Party G-03 (Lt. F. W. Rossmann, project director), R. W. Anderson, A. D. Harrison, J. D. Love, R. C. Philipp, T. D. Salisbury, M. B. White (space and physical geodesy branch), R. L. Taylor (geodetic advisor, state of Florida), E. E. Carlson (horizontal network branch), and E. I. Balazs (vertical network branch).

\section{Appendix I. Parameters Involved in Similarity TRANSFORMATIONS}

The seven-parameter (similiarity) transformation between any two Cartesian systems, e.g., from $(\mathbf{u}, \mathbf{v}, \mathbf{w})$ to $(\mathbf{x}, \mathbf{y}, \mathbf{z})$, or in short $(\mathbf{u}, \mathbf{v}, \mathbf{w}) \rightarrow(\mathbf{x}, \mathbf{y}, \mathbf{z})$ can be written

$$
\left\{\begin{array}{l}
\mathbf{x} \\
\mathbf{y} \\
\mathbf{z}
\end{array}\right\}=\left\{\begin{array}{l}
\Delta \mathbf{x} \\
\Delta \mathbf{y} \\
\Delta \mathbf{z}
\end{array}\right\}+(1+\delta s)\left[\begin{array}{ccc}
1 & \delta \omega & -\delta \psi \\
-\delta \omega & 1 & \delta \epsilon \\
\delta \psi & -\delta \epsilon & 1
\end{array}\right]\left\{\begin{array}{c}
\mathbf{u} \\
\mathbf{v} \\
\mathbf{w}
\end{array}\right\} \ldots \ldots \ldots \ldots \ldots \ldots
$$

where $\Delta \mathbf{x}, \Delta \mathbf{y}$, and $\Delta \mathbf{z}=$ coordinates of the origin of the frame $(\mathbf{u}, \mathbf{v}, \mathbf{w})$ in the frame $(\mathbf{x}, \mathbf{y}, \mathbf{z}) ; \delta \epsilon, \delta \psi, \delta \omega=$ differential rotations (expressed in radians), respectively, around the axes $(\mathbf{u}, \mathbf{v}, \mathbf{w})$ to establish parallelism with the $(\mathbf{x}, \mathbf{y}, \mathbf{z})$ frame. (Positive rotations are counterclockwise rotations as viewed looking toward the origin of the right-handed coordinate system); and $\delta s=$ differential scale change (expressed in $\mathrm{ppm} \times 10^{-6}$ ) (see Table 2 ).

\section{ApPendix II. References}

Bodnar, A. N. (1990a). “A high-resolution geoid for the United States." EOS Trans. Amer. Geophysical Union, 71(46), 1,793.

Bodnar, A. N. (1990b). "National geodetic reference system. Statewide upgrade policy." Proc. ACSMS-ASPRS Fall Conv., American Congress on Surveying and Mapping, Nov. 5-10.

Burton, L. L., Jr. (1989). "Analysis of the county level land information system in the state of Florida: User trends and perceived accuracy requirements." Proc. GIS/ LIS' 89, American Congress on Surveying and Mapping, Nov. 26-30, 348-366.

Craymer, M. R., Wells, D. E. Vaníček, P., and Devlin, R. I. (1990). "Specifications for urban GPS surveys." Surveying and Land Information Systems, 50(4), 251259.

Ethridge, M. M. (1989). "Does the National Geodetic Reference System need to be upgraded?" Point of Beginning Magazine, 15(1), 26, 30-31.

Geometric geodetic accuracy standards and specifications for using GPS relative positioning techniques. Version 5.0. (1988). Federal Geodetic Control Committee, Nat. Geodetic Information Branch, Nat. Oceanic and Atmospheric Administration, Rockville, Md.

Hajela, D. (1990). "Obtaining centimeter-precision heights by GPS observations over small areas." GPS World, 1(1), 55-59.

Mader, G. L., Schenewerk, M. S., and Chin, M. M. (1990). OMNI 1.00. users guide. National Geodetic Information Branch, Nat. Oceanic and Atmospheric Administration, Rockville, Md.

McCarthy, D. D. (1989). "IERS standards (1989)." IERS Technical Note 3, Central Bureau of Int. Earth Rotation Service, Observatoire de Paris, Paris, France.

"NEOS. The U.S. National Earth Orientation Service." (1990). National Geodetic Information Branch, NOAA, Rockville, Md.

Rapp, R. H., and Pavlis, N. K. (1990). "The development and analysis of geopotential coefficient models to spherical harmonic degree 360." J. Geophysical Res., 95, Dec., 21,885-21,911. 
Schwarz, C. R., and Wade, E. B. (1990). "The North American Datum of 1983: Project methodology and execution." Bulletin Géodésique, 64(1), 28-62.

Snay, R. A. (1989). "Accuracy analysis." North American Datum of 1983, C. R. Schwarz, ed, National Geodetic Information Branch, Nat. Oceanic and Atmospheric Administration, Rockville, Md.

Snay, R. A. (1990). "Accuracy analysis for the NAD 83 Geodetic Reference System." Bulletin Géodésique, 64(1), 1-27.

Soler, T., and Hothem, L. D. (1989). "Important parameters used in geodetic transformations." J. Surv. Engrg., ASCE, 115(4), 414-417.

Standards and specifications for geodetic control networks. (1984). Federal Geodetic Control Committee, Nat. Geodetic Information Branch, Nat. Oceanic and Atmospheric Administration, Rockville, Md.

Strange, W. E. (1990). "Overview of NGS high accuracy GPS network activities in North America." EOS, Trans. Amer. Geophysical Union, 71(17), 417.

Taylor, R. L. (1989). "Florida's high precision geodetic network-an update." Backsights and Forsights, Fourth Quarter, 11-19. 